REVIEW

\title{
The nucleolar structure and nucleolar proteins as indicators of cell proliferation events in plants
}

\author{
Fernando González-Camacho and Francisco Javier Medina \\ Centro de Investigaciones Biológicas (CSIC), Madrid, Spain
}

Received $16^{\text {th }}$ July 2005.

Published online $5^{\text {th }}$ October 2005.

\begin{abstract}
Summary
Cell proliferation is a crucial cellular process which influences development. In plants, meristems are formed by actively proliferating cells, in which the main expression of proliferation is the existence of a cell division cycle. Many cell activities are influenced by the cell proliferation status and cell cycle progression, among them ribosome biogenesis, which is morphologically expressed as the nucleolus. The connection is established through nucleolar proteins, which regulate the synthesis and processing of preribosomal precursors and, at the same time, are targets of various cell cycle regulators, such as certain kinases. Nucleolin is one of these nucleolar proteins, whose level increases with cell proliferation and depends on the cell cycle stages. Not only the levels, but also other important features of the protein, such as its distribution in situ in the nucleolus, its phosphorylation and its physiological degradation, depend on these parameters. Furthermore, since the nucleolar structure is highly sensitive to functional variations, distinct nucleolar structures, regarding the nucleolar size and the distribution of nucleolar subcomponents, have been defined for each period of the cell cycle, using synchronized cells. In addition to increasing our knowledge of cellular physiology, these relationships can be used to mark the proliferative state of the cell and the periods of cell cycle.
\end{abstract}

Keywords: ribosome - cell cycle - nucleolin - fibrillarin - Western blotting - electron microscopy

\section{CELL PROLIFERATION IN PLANT MERISTEMS}

The anatomy of adult higher plants comprises two distal meristems, namely the primary shoot meristem and the primary root meristem, composed

Francisco Javier Medina, Centro de Investigaciones Biológicas (CSIC), Ramiro de Maeztu 9, E-28040 Madrid, Spain

品 fjmedina@cib.csic.es

용+34918373112 ext. 4261

回 +34915360432 of small populations of highly proliferating cells whose function is to continuously provide new cells for plant growth and development. In particular, shoot meristem gives rise to leaves, stems and floral organs, and the primary root meristem elaborates the primary root, while secondary roots are initiated within the pericycle at some distance distal from the root tip. Therefore, meristems play a crucial role in development patterning and morphogenesis (Dolan et al. 1993).

At the cellular level, the main consequence of cell proliferation is the establishment and progression of the cell cycle, composed of successive phases in which a mother cell distributes its genetic load equally between two daughter cells, and then DNA is replicated in each of these cells to 
qualify them to be mother cells in the next division. However, there is increasing evidence not only that the cell cycle serves for assuring cell division or for making possible cell growth, but cell cycling activity and regulation have a strong influence on the definition of the developmental patterns of the plant (Beemster et al. 2003, Gutierrez 2005).

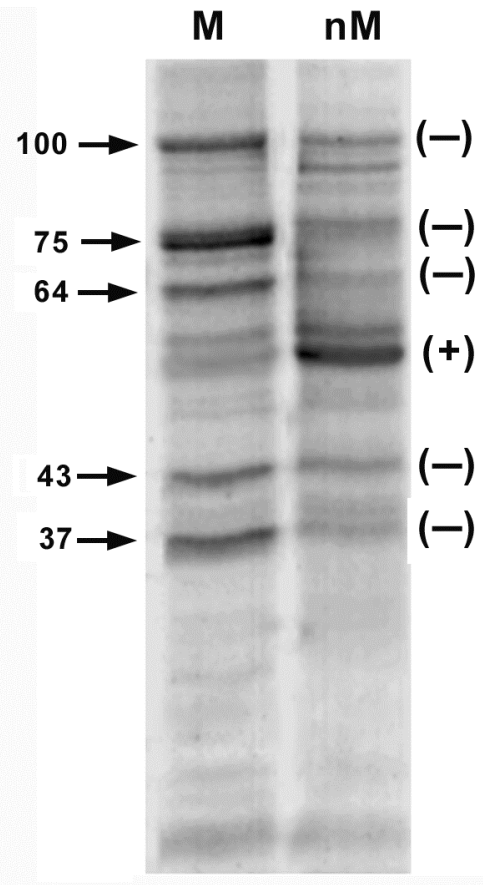

A

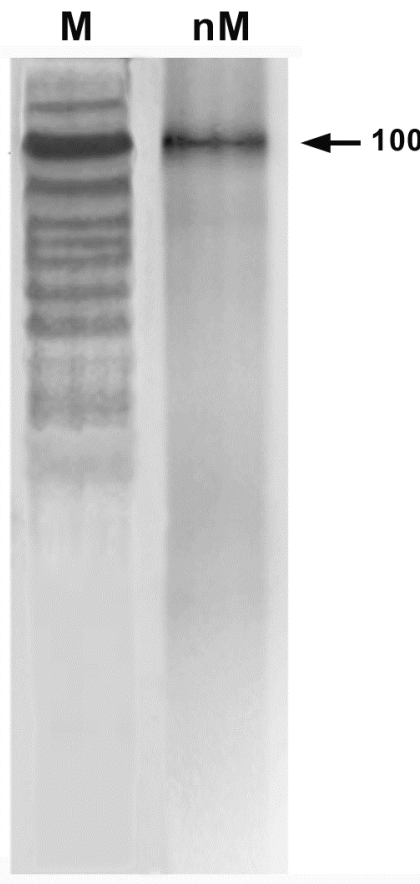

B

Fig. 1.- (A) Soluble nuclear proteins from onion root cells separated by SDS-PAGE and stained with Coomassie brilliant blue. M: meristematic (highly proliferating) cells. nM: non-meristematic (non-proliferating) cells. Arrows and numbers at the left indicate the molecular weights in $\mathrm{kDa}$ of the major bands of the meristematic population. All these bands are quantitatively reduced in the non-meristematic population (-), but a band around $55 \mathrm{kDa}$ is enhanced (+). (B) Western blotting of the same extracts probed with an antibody raised against the onion nucleolin-like protein. The reaction is much more intense in the meristematic population (lane $\mathrm{M}$ ), in which the antibody recognizes a $100 \mathrm{kDa}$ major band and some other minor bands which are the product of its physiological degradation. In non-meristematic cells, the $100 \mathrm{kDa}$ band is more weakly revealed and it is not accompanied by other bands.

\section{CELL PROLIFERATION AND RIBOSOME BIOGENESIS}

Cell cycling activity in proliferating cells encompasses an intense and continuous production of new cellular materials. Therefore, the cellular mechanisms regulating cell growth and proliferation and, consequently, cell cycle progression, affect the regulation of all basic cellular processes to a greater or lesser degree. In particular, there is much experimental evidence of the relationship of cell proliferation events with ribosome biogenesis, a process which is a key in cellular physiology since ribosomes are the cellular factories in which proteins are synthesized. Ribosome biogenesis is morphologically expressed in the cell nucleus as a prominent organelle, the nucleolus, which is the best known visible structure associated with expression of a definite set of genes (i.e. ribosomal genes or rRNA genes), which are clustered in a particular chromosomal site; the nucleolar organizing region (NOR).

Factors stimulating cell growth and division produce an increase of the rate of ribosome biogenesis, and this rate is regulated during the cell cycle, in which it increases from G1 to G2 and is stopped during mitosis, accompanied by the disassembly of the nucleolus (Kwiatkowska and Maszewski 1979, Klein and Grummt 1999, Hernandez-Verdun and Roussel 2003). 


\section{CELL PROLIFERATION, CELL CYCLE AND NUCLEOLAR PROTEINS}

The evolution of the nucleolus during cell cycle periods is associated with changes detected in nucleolar proteins. These proteins, acting as targets of factors controlling cell proliferation and cell cycle progression, and, at the same time, as regulators of the rate of pre-rRNA transcription and/or processing at different levels, could be the bridge connecting cell cycle and proliferation events and regulation of ribosome biogenesis (Olson 1991, Medina and González-Camacho 2003, Volkov et al. 2004).

Plant proliferating cells show a distinct pattern of expressed proteins with respect to nonproliferating cells. This was evidenced in the onion, using the extract of nuclear proteins which are soluble in a low ionic strength buffer. This extract is enriched in ribonucleoproteins, active in nuclear RNA metabolism (Busch et al. 1978, Bugler et al. 1982). The electrophoretic pattern showed outstanding differences between the extracts obtained from cells differing in their proliferative state, with five major proteins enriched in proliferating cells, whose levels were decreased in differentiated cells (Fig. 1A). The separation of the same extracts, in two-dimensional gels confirmed these differences (González-Camacho and Medina 2004).

Among nucleolar proteins, nucleolin is a conserved protein, first described in mammalian cells, and also characterized in other animals, in plants and in yeast, in which the homologous proteins are called "nucleolin-like" proteins. In all cases it has been defined as a multifunctional protein, known to play roles in determining the structure of the chromatin containing the ribosomal RNA (rRNA) genes, in the activation of rRNA gene transcription, and in different steps of prerRNA processing (Tuteja and Tuteja 1998, Ginisty et al. 1999). At least part of these functions are determined by the level of phosphorylation of nucleolin by two kinases, namely cdc2 kinase and casein kinase II, known to play crucial roles in the regulation and co-ordination of cellular events involved in cell cycle and proliferation. In fact, these two kinases are known to phosphorylate a battery of different targets (among which is nucleolin) as a response to proliferative signals, and as a modulation of the different periods of the cell cycle (Dorée and Galas 1994, Riera et al. 2001). As a consequence, nucleolin is one of the most abundant proteins in proliferating cells, whereas its expression is greatly reduced in non-proliferating (differentiated or quiescent) cells (Srivastava and Pollard 1999, Medina et al. 2001). This can be evidenced after observing the different signal revealed by an antibody raised against the onion nucleolin-like protein on nuclear soluble extracts obtained proliferating and non-proliferating cells (Fig. 1B).

Furthermore, the levels of nucleolin have been shown to be highly dependant on the phase of the cell cycle. In both mammalian and plant cells, these levels are minimal at the G1 stage, they increase at a high rate up to $\mathrm{S}$ period, and then the increase is slower, but sustained, until the peak level is reached at G2 stage, immediately before mitosis (De Cárcer et al. 1997, Sirri et al. 1997). During mitosis, nucleolin was localized in the chromosomal nucleolar organizer domain, in a perichromosomal ribonucleoprotein sheath and in prenucleolar bodies at telophase, where it partially colocalizes with RNA polymerase I and with the transcription factor UBF, expressing the postmitotic resumption of the nucleolar activity (Ochs et al. 1983, Jiménez-García et al. 1989, Medina et al. 1995, Medina et al. 2001, De Cárcer et al. 1997, Medina et al., unpublished results). In alfalfa, the nucleolin gene is not expressed in mitotic-arrested cells, but, upon mitogenic stimulation and cell cycle reentry, the gene is induced very early, exactly at the same time as a marker cyclin gene, indicating that nucleolin gene expression and cyclin expression could be regulated by the same factors. Otherwise, the expression of nucleolin is restricted to proliferating tissues of the plant (Bögre et al. 1996). In peas, the expression of nucleolin is regulated by light, following a close correlation with the effects of light in increasing cell proliferation and mitotic activity (Reichler et al. 2001).

However, it is not only the expression of nucleolin that has been shown to be dependent on the cell proliferation status and of cell cycle progression, but some features of the protein are also affected by these cellular factors. Among these features, phosphorylation is a post-translational modification of nucleolin which is linked with cell proliferation. Two-dimensional Western blots showed that the number of isoforms of the onion nucleolin-like protein, as well as the range of isoelectric point of these isoforms, was markedly larger in meristematic highly proliferating cells than in differentiated nonproliferating cells. A dephosphorylation assay on the meristematic cell population evidenced that the differential amount of phosphate groups of the protein was the cause of the differences (González-Camacho and Medina 2004).

The localization of the protein in the nucleolus is another feature of the protein dependent on the cell proliferation status. In proliferating cells, nucleolin has a definite subnucleolar location in the region of the dense fibrillar component immediately surrounding fibrillar centers. This preferential localization is lost in quiescent cells, which do not show a localized distribution of the protein in their nucleoli (Medina et al. 2001). 
Finally, a process of physiological endoproteolytic maturation was described in mammalian nucleolin, associated with the functioning of the protein in the regulation of the synthesis and processing of preribosomal precursors (Bouche et al. 1984, Fang and Yeh
1993). This physiological proteolysis is another post-translational modification of nucleolin also related to cell cycle progression, as has been shown in onion cells (Medina et al., unpublished results).

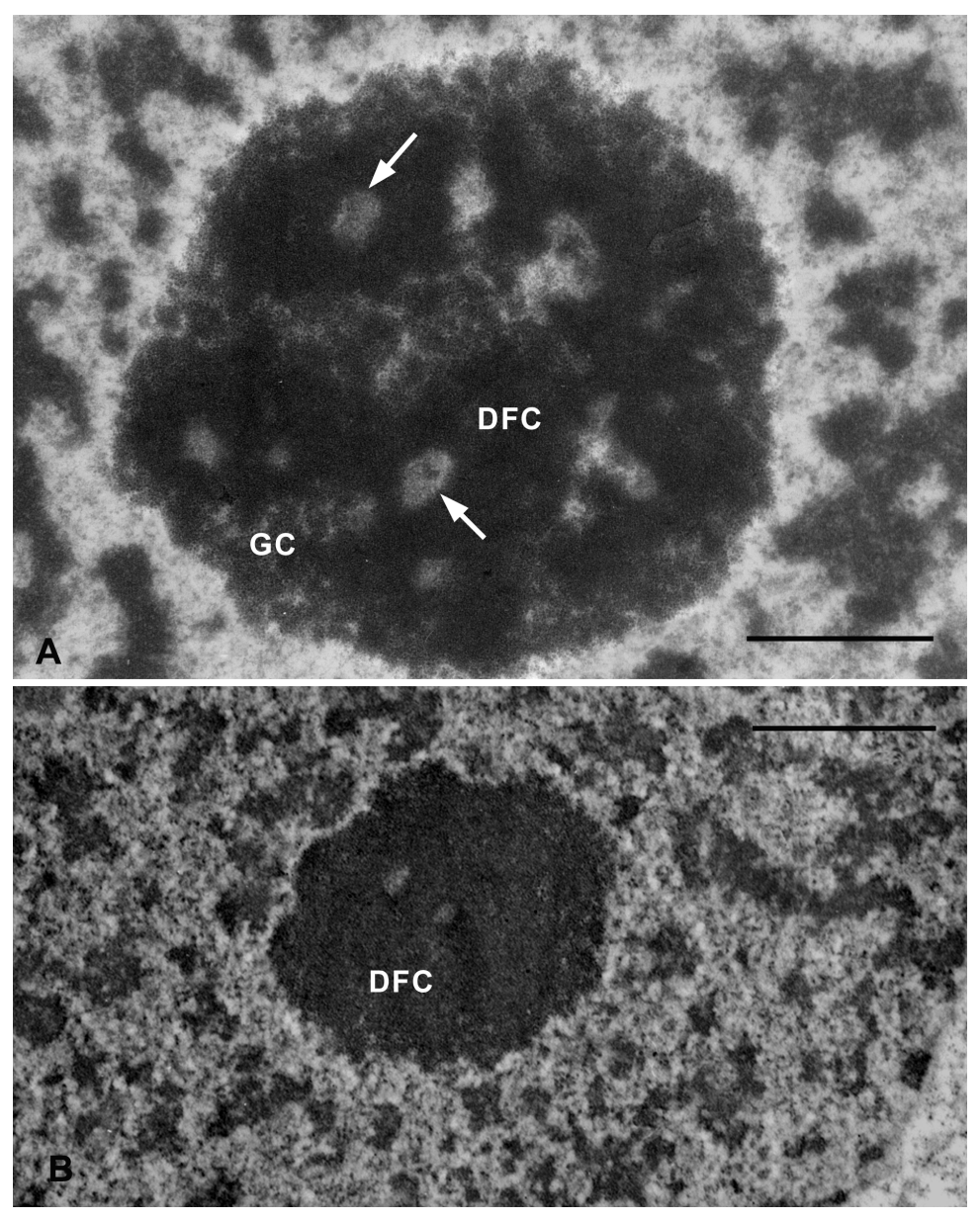

Fig. 2. Electron microscopical images of nucleoli from an onion root meristematic cells (A) and from a nonmeristematic root cell (B). The structural differences are outstanding. The proliferating cell contains abundant granular component (GC), which is intermingled with the masses of dense fibrillar component (DFC), appearing in sections surrounding them. Fibrillar centers (mostly homogeneous; arrows) are embedded into the DFC masses. On the contrary, the nucleolus of the differentiated root cell is much smaller and is only formed by dense fibrillar component (DFC). Bars indicate $1 \mu \mathrm{m}$.

Another nucleolar protein, fibrillarin, was identified in plants as homologous to the protein which had been previously characterized in mammals and yeast (Guiltinan et al. 1988, Cerdido and Medina 1995). Studies on the fibrillarin gene after its molecular cloning in Arabidopsis, have demonstrated that its expression is regulated by hormones, such as abscisic acid, and it is also modulated by aging (Pih et al. 2000).

The levels of fibrillarin have been shown to alter depending on cell cycle progression and on the differentiation state of the cell. Thus, similar to the results with nucleolin, differentiated root cells showed lower levels of fibrillarin than meristematic highly proliferating root cells when proteins separated from the same amount of nuclei from the two tissues were probed with an anti-fibrillarin antiserum in a Western blotting experiment. Throughout the cell cycle, the variation in the levels of fibrillarin was quantitatively measured, showing that labeling of G2 nucleoli was more than double than that of G1 nucleoli (Cerdido and 
Medina 1995). Furthermore, a flow cytometry study, performed on isolated nuclei throughout interphase, revealed that fibrillarin expression was low during the G1 period and progressively increased at a high rate until the $\mathrm{S}$ period; later, in $\mathrm{G} 2$, this expression continued increasing, but at a lower rate, so that the maximum level was reached just before mitosis (Medina et al. 2000).
All these facts and features make these nucleolar proteins suitable and highly valuable markers for studying the influence on ribosome biogenesis of mechanisms controlling the cell cycle and proliferation events in plants.

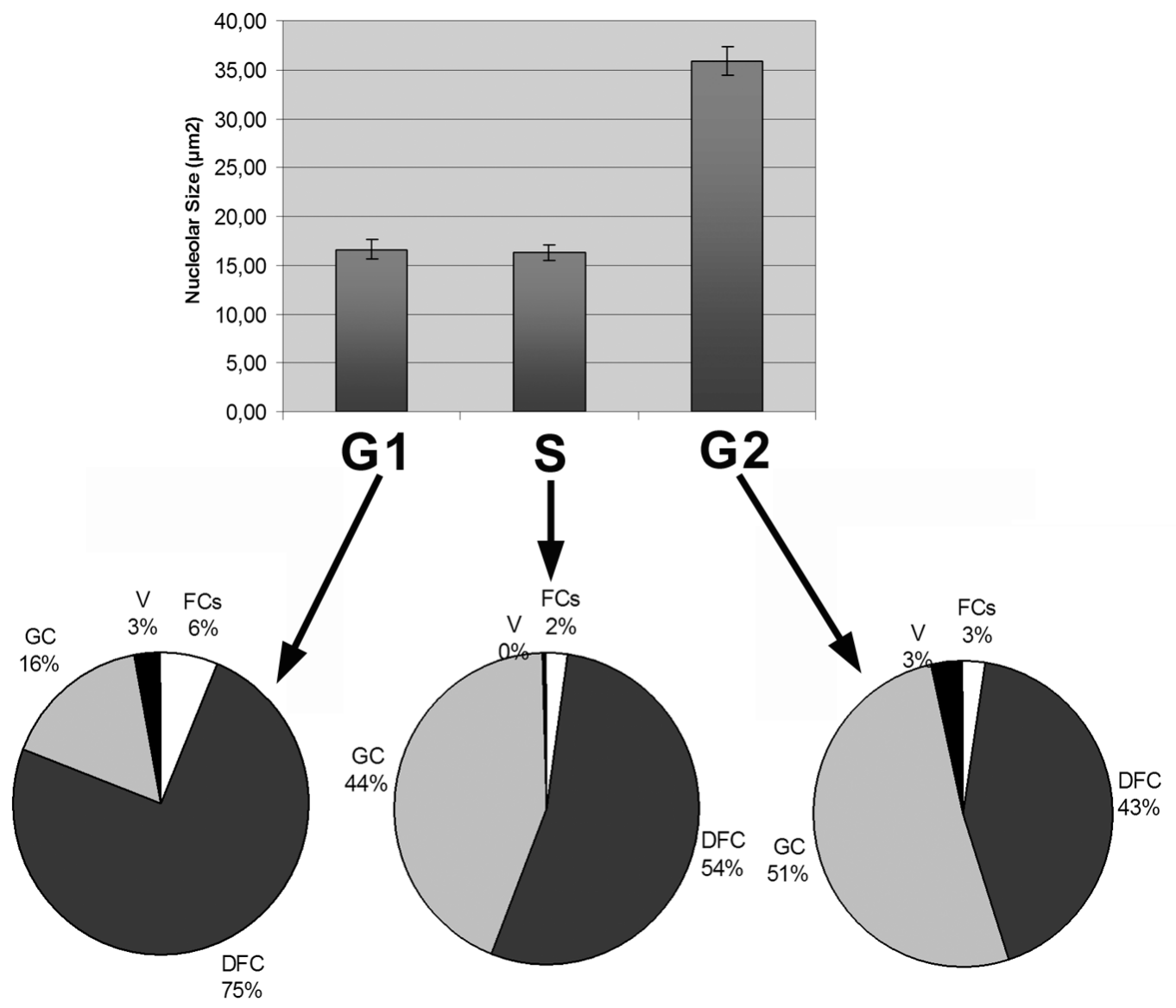

Fig. 3. Quantitative study of the nucleolar size and the distribution of nucleolar subcomponents throughout the successive periods of the interphase of the cell cycle, in onion root meristematic cells synchronized with hydroxyurea. The nucleolar size remains constant through G1 and S periods and is doubled in G2. The relative contribution of the dense fibrillar component (DFC) to the nucleolar volume decreases through interphase at the expenses of the increase of the granular component.

\section{CELL PROLIFERATION, CELL CYCLE AND NUCLEOLAR STRUCTURE}

The nucleolar morphology is constituted by a few structural components, namely fibrillar centers, a dense fibrillar component and a granular component, whose organization and quantitative distribution are highly dependent on the physiological state of the cell, on the cellular proliferating activity and on the different periods of the cell cycle (Risueño and Medina 1986, Junéra et al. 1995, Medina et al. 2000). Since the nucleolar structure is highly sensitive to functional alterations, the ultrastructure of the nucleolus of an actively proliferating cell shows dramatic changes with respect to the nucleolar morphology exhibited by a differentiated or non-proliferating cell (Fig. 2). In plants, meristematic cell nucleoli are characterized by a large size and an abundant granular component, which appears intermingled with the dense fibrillar component. In ultrathin sections, this dense fibrillar component is 
organized as apparently separated territories, surrounded by the granular component and containing fibrillar centers in their interior (Fig. 2A). In contrast, the size of the nucleoli of nonproliferating cells is much smaller and they are constituted practically exclusively by a dense fibrillar component. (Fig. 2B).

Nucleolar fibrillar centers have been particularly studied in their ultrastructure and morphometric features (number and size) in relation to nucleolar activity. In general, it has been observed that a high nucleolar activity is characterized by a multiplicity of fibrillar centers, which are of small size. In contrast, low active nucleoli contain few fibrillar centers of larger size (Jordan and McGovern 1981, Medina et al. 1983). Furthermore, in plant cells, two structural types of fibrillar centers were defined, namely homogeneous and heterogeneous fibrillar centers. Both structural types share the presence of proteins and decondensed chromatin fibers as constituents, but the second type contains in addition small inclusions of condensed chromatin. The appearance of heterogeneous fibrillar centers is linked to the states of low functional activity in the nucleolus, including their presence in quiescent cells (Risueño et al. 1982).

Other nucleolar components which have been placed in relation to nucleolar activity were the socalled nucleolar "vacuoles", large intranucleolar territories with a loose organization of fibrils and granules. The appearance of these "vacuoles" depends on the functional state of the nucleolus, being particularly associated with an enhanced activity in transcription and processing of preribosomal precursors (Moreno Díaz de la Espina et al. 1980).

In view of the close relationship between different nucleolar structural parameters and the functional activity of ribosome biogenesis, and taking into account that the cellular functional process is strictly regulated according to cell cycle progression, a systematic study was undertaken in plant cells with the purpose of assigning definite nucleolar structural patterns and morphometrical features of the nucleolus and nucleolar subcomponents to the successive cell cycle phases. For this purpose, onion root meristematic cells, synchronized in their cell cycle progression by a treatment with hydroxyurea, were used. The results indicate that the nucleolar size remains practically unchanged from the postmitotic nucleologenesis till the end of the $\mathrm{S}$ period, although a redistribution of subcomponents can be appreciated from the G1 to the $\mathrm{S}$ period, consisting of an increase of the granular component at the expenses of the diminution of the dense fibrillar component; fibrillar centers are also reduced in size. In G2, a dramatic enlargement of the nucleolus takes place, the size reaching double magnitude with respect to the preceding periods. This increase in size is mostly due to the production of the granular component, whose relative contribution to the nucleolar volume is still increased, whereas the dense fibrillar component is reduced in relative terms (Fig. 3).

Therefore, we can conclude that there is, in fact, a pattern of nucleolar organization associated with each period of the cell cycle. Interestingly, nucleolar activity is closely and strictly related to cell cycle progression in plant proliferating cells, and this relationship may become evident and can be evaluated by observing the nucleolar structure and the levels and features of certain nucleolar proteins. These parameters can be used as markers of the cellular proliferative status and of the course of cell cycle progression.

\section{ACKNOWLEDGEMENT}

Work performed in the authors' laboratory was supported by Grants from the Spanish "Plan Nacional de Investigación Científica y Desarrollo Tecnológico" Ref. Nos. ESP2001-4522-PE, and ESP2003-09475-C02-02.

\section{REFERENCES}

Beemster G.T.S., Fiorani F., Inzé D.: Cell cycle: the key to plant growth control? Trends Plant Sci. 8:154-158, 2003.

Bögre L., Jonak C., Mink M. et al.: Developmental and cell cycle regulation of alfalfa nucMs 1 , a plant homolog of the yeast Nsr1 and mammalian nucleolin. Plant Cell 8:417-428, 1996.

Bouche G., Caizergues-Ferrer M., Bugler B., Amalric F.: Interrelations between the maturation of a $100 \mathrm{KDa}$ nucleolar protein and pre-rRNA synthesis in $\mathrm{CHO}$ cells. Nucl. Acids Res. 12:3025-3035, 1984.

Bugler B., Caizergues-Ferrer M., Bouche G., Bourbon H.M., Amalric F.: Detection and localization of a class of proteins immunologically related to a $100-\mathrm{KDa}$ nucleolar protein. Eur. J. Biochem. 128:475480, 1982.

Busch H., Ballal N.R., Rao M.R.S., Choi Y.C., Rothblum L.I.: Factors affecting nucleolar rDNA readouts. In Busch, H. (ed.): The Cell Nucleus, vol. 5, Chromatin, part B. Academic Press, New York, San Francisco, London 1978, pp.416-468.

Cerdido A., Medina F.J.: Subnucleolar location of fibrillarin and variation in its levels during the 
cell cycle and during differentiation of plant cells. Chromosoma 103:625-634, 1995.

De Cárcer G., Cerdido A., Medina F.J.: NopA64, a novel nucleolar phosphoprotein from proliferating onion cells, sharing immunological determinants with mammalian nucleolin. Planta 201:487-495, 1997.

Dolan L., Janmaat K., Willemsen V. et al.: Cellular organisation of the Arabidopsis thaliana root. Development 119:71-84, 1993.

Dorée M., Galas S.: The cyclin-dependent protein kinases and the control of cell division. FASEB J. 8:1114-1121, 1994.

Fang S.H., Yeh N.H.: The self-cleaving activity of nucleolin determines its molecular dynamics in relation to cell proliferation. Exp. Cell Res. 208:48-53, 1993.

Ginisty H., Sicard H., Roger B., Bouvet P.: Structure and functions of nucleolin. J. Cell Sci. 112:761-772, 1999.

González-Camacho F., Medina F.J.: Identification of specific plant nucleolar phosphoproteins in a functional proteomic analysis. Proteomics 4:407-417, 2004.

Guiltinan M.J., Schelling M.E., Ehtesham N.Z., Thomas J.C., Christensen M.E.: The nucleolar RNA binding protein B-36 is highly conserved among plants. Eur. J. Cell Biol. 46:547-553, 1988.

Gutierrez C.: Coupling cell proliferation and development in plants. Nature Cell Biol. 7:535-541, 2005

Hernandez-Verdun D., Roussel P.: Regulators of nucleolar functions. Prog. Cell Cycle Res. 5:301-308, 2003.

Jiménez-García L.F., Rothblum L.I., Busch H., Ochs R.L.: Nucleologenesis: use of nonisotopic "in situ" hybridization and immunocytochemistry to compare the localization of rDNA and nucleolar proteins during mitosis. Biol. Cell 65:239-246, 1989.

Jordan E.G., McGovern J.H.: The quantitative relationship of the fibrillar centres and other nucleolar components to changes in growth conditions, serum deprivation and low doses of actinomycin D in cultured diploid human fibroblasts (strain MRC-5). J. Cell Sci. 52:373-389, 1981.

Junéra H.R., Masson C., Géraud G., HernandezVerdun D.: The three-dimensional organization of ribosomal genes and the architecture of the nucleoli vary with $\mathrm{G}_{1}, \mathrm{~S}$ and $\mathrm{G}_{2}$ phases. J. Cell Sci. 108:3427-3441, 1995.

Klein J., Grummt I.: Cell cycle-dependent regulation of RNA polymerase I transcription: the nucleolar transcription factor UBF is inactive in mitosis and early G1. Proc. Natl. Acad. Sci. USA 96:6096-6101, 1999.

Kwiatkowska M., Maszewski J.: Changes in the activity of RNA polymerase detected in situ and the intensity of $3 \mathrm{H}$ uridine incorporation into the nucleolus and the nucleus of interphase cells in antheridial filaments of Chara vulgaris L. Folia Histochem. Cytochem. 17:275-286, 1979.

Medina F.J., Risueño M.C., Moreno Díaz de la Espina S.: 3-D Reconstruction and morphometry of fibrillar centres in plant cells in relation to nucleolar activity. Biol. Cell 48:31-38, 1983.

Medina F.J., Cerdido A., Fernández-Gómez M.E.: Components of the nucleolar processing complex (pre-rRNA, fibrillarin and nucleolin) colocalize during mitosis and are incorporated to daughter cell nucleoli. Exp. Cell Res. 221:111-125, 1995.

Medina F.J., Cerdido A., De Cárcer G.: The functional organization of the nucleolus in proliferating plant cells. Eur. J. Histochem. 44:117-131, 2000.

Medina F.J., González-Camacho F., Cerdido A., De Cárcer G.: In situ localization of the onion nucleolar protein NopA64 is dependent on cell proliferation mechanisms and cell cycle phases. In Dini, L. and M. Catalano (eds.): Proc. 5th Multinational Congress on Electron Microscopy. Rinton Press Inc., Princeton, New Jersey. 2001, pp. 197-207.

Medina F.J., González-Camacho F.: Nucleolar proteins and cell proliferation in plant cells. Recent Res. Devel. Plant Biol. 3:55-68, 2003.

Moreno Díaz de la Espina S., Medina F.J., Risueño M.C.: Correlation of nucleolar activity and nucleolar vacuolation in plant cells. Eur. J. Cell Biol. 22:724-729, 1980.

Ochs R.L., Lischwe M.A., O'Leary P., Busch H.: Localization of nucleolar phosphoproteins B23 and C23 during mitosis. Exp. Cell Res. 146:139-149, 1983.

Olson M.O.J.: The role of proteins in nucleolar structure and function. In Strauss, P.R. and S.H. Wilson (eds.): The Eukaryotic Nucleus. Molecular Biochemistry and Macromolecular Assemblies. The Telford Press, Caldwell, New Jersey 1991, pp. 519-559.

Pih K.T., Yi M.J., Liang Y.S. et al.: Molecular cloning and targeting of a fibrillarin homolog from Arabidopsis. Plant Physiol. 123: 51-58, 2000.

Reichler S.A., Balk J., Brown M.E. et al.: Light differentially regulates cell division and the mRNA abundance of pea nucleolin during deetiolation. Plant Physiol. 125:339-350, 2001.

Riera M., Peracchia G., Pagès M.: Distinctive features of plant protein kinase CK2. Mol. Cell Biochem. 227:119-127, 2001.

Risueño M.C., Medina F.J.: The nucleolar structure in plant cells. Cell Biol. Rews. (RBC) 7:1-154, 1986. 
Risueño M.C., Medina F.J., Moreno Díaz de la Espina S.: Nucleolar fibrillar centres in plant meristematic cells: ultrastructure, cytochemistry and autoradiography. J. Cell Sci. 58:313-329, 1982.

Sirri V., Roussel P., Gendron M.C., HernandezVerdun D.: Amount of the two major AgNOR proteins, nucleolin and protein B23 is cellcycle dependent. Cytometry 28:147-156, 1997.

Srivastava M., Pollard H.B.: Molecular dissection of nucleolin's role in growth and cell proliferation: new insights. FASEB J. 13:1911-1922, 1999.

Tuteja R., Tuteja N.: Nucleolin: a multifunctional major nucleolar protein. CRC Crit. Rev. Biochem. Mol. Biol. 33:407-436, 1998.

Volkov R.A., Medina F.J., Zentgraf U., Hemleben V.: Organization and molecular evolution of rDNA, nucleolar dominance, and nucleolus structure. Prog. Botany 65:106-146, 2004. 